\title{
ANTIOXIDATIVE ACTIVITY OF ALPHA-MANGOSTIN IN ACETALDEHYDE-INDUCED HEPATIC STELLATE CELLS: AN IN VITRO STUDY
}

\author{
NOVRIANTIKA LESTARI ${ }^{1,2}$, SAMUEL PRATAMA3 ${ }^{3}$ KELVIN THEANDRO GOTAMA ${ }^{3}$, VIVIAN SOETIKNO4, \\ MELVA LOUISA $^{4 *}$
}

${ }^{1}$ Master Program in Biomedical Sciences, Faculty of Medicine, Universitas Indonesia, ${ }^{2}$ Department of Pharmacology, Faculty of Medicine and Health Science, University of Bengkulu, ${ }^{3}$ Faculty of Medicine, Universitas Indonesia, Jakarta, 10430, Indonesia, ${ }^{4}$ Department of Pharmacology and Therapeutics, Faculty of Medicine, Universitas Indonesia. Email: melva.louisa@gmail.com

Received: 20 August 2018, Revised and Accepted: 18 February 2019

ABSTRACT

Objective: Alcohol accumulation in the liver can cause pathological disorders such as liver fibrosis that can develop into hepatocellular carcinoma, one of the main causes of mortality associated with liver disease. The previous studies have shown that a plant compound, alpha-mangostin, has an antioxidant effect in the inhibition of pancreatic tumor growth in vitro. This study aimed to analyze the antioxidative properties of alpha-mangostin in acetaldehyde-induced liver fibrosis in vitro.

Methods: Immortalized hepatic stellate cells (HSCs), of the LX-2 cell line, were incubated with acetaldehyde in the presence or absence of alphamangostin $(10$ and $20 \mu \mathrm{M})$. The cells were then counted and lysed, and LX-2 cell viability was determined with the trypan blue exclusion method. The malondialdehyde levels, superoxide dismutase activity, and glutathione (GSH) levels were also determined using the cell lysates.

Results: Acetaldehyde treatment resulted in a significant increase in HSC cell viability and decreased the production of GSH. Alpha-mangostin treatment resulted in reduced cell viability of the HSCs and prevention of the loss of intracellular GSH.

Conclusion: Alpha-mangostin reduced acetaldehyde-induced cell proliferation, and this was affected at least in part by its antioxidative properties.

Keywords: Acetaldehyde, Alpha-mangostin, Antioxidant.

(c) 2019 The Authors. Published by Innovare Academic Sciences Pvt Ltd. This is an open access article under the CC BY license (http://creativecommons. org/licenses/by/4. 0/) DOI: http://dx.doi.org/10.22159/ijap.2019.v11s1.16089

\section{INTRODUCTION}

Alcohol accumulation in the liver can cause pathological disorders such as liver fibrosis. This disease can develop into hepatocellular carcinoma, which is one of the main causes of mortality associated with liver disease [1,2]. Hepatic stellate cells (HSCs) are central to the development of fibrosis following alcohol accumulation. In the normal liver, HSCs are quiescent cells that differentiate into myofibroblasts when activated. Other than alcohol, HSC can be activated by toxins, disorders of immune systems, or viral and parasitic infections. Active HSCs are characterized by increased proliferation, contractility, and secretion of the fibrogenesis mediator, transforming growth factor $\beta$ (TGF- $\beta$ ) [3-5].

Alcohol is metabolized in the liver by alcohol dehydrogenase to produce acetaldehyde, a toxic substance, which further metabolized to form acetate, a less active byproduct. When there is a large amount of alcohol, CYP2E1 will also active and metabolize alcohol to acetaldehyde and further produce acetate and reactive oxygen species (ROS) [6,7]. ROS enhance HSC activation and play an important role in the fibrogenesis that occurs in alcohol-induced liver disease. TGF- $\beta$ increases the production of ROS and suppresses the production of antioxidants. ROS, in turn, activates latent TGF- $\beta$ and induces its expression. Consequently, TGF- $\beta$ acts synergistically with alcohol in inducing oxidative stress, thus increasing alcohol-induced liver damage $[8,9]$.

There is no standard therapy for the treatment of liver fibrosis. Research on the treatment of liver fibrosis using active compounds from plants is still ongoing. Alpha-mangostin, one of the main xanthones from Garcinia mangostana, is known to have anti-inflammatory, antioxidant, cardioprotective, antidiabetic, and anticancer properties [10,11]. In a previous study by Rahmaniah et al., alpha-mangostin was shown to decrease the ratios of pSmad/Smad and pAkt/Akt in an in vitro liver fibrosis model induced by TGF- $\beta$ [12]. Further, investigations of the mechanism of the action of alpha-mangostin as a hepatoprotector are still required. This study aimed to analyze the antioxidant activity of alpha-mangostin in a model of acetaldehyde-induced liver fibrosis.

\section{METHODS}

Human HSCs, of the LX-2 cell line, were purchased from Millipore (USA; Cat. No. SCC064). Alpha-mangostin was obtained from Santa Cruz Biotechnology (Texas, USA). Acetaldehyde and dimethylsulfoxide (DMSO) were purchased from Sigma-Aldrich (Singapore). Dulbecco's modified Eagle's Medium (DMEM) high glucose-supplemented media, heat-inactivated fetal bovine serum (FBS), penicillin, streptomycin, and Fungizone were obtained from Biowest (USA). Thermo Scientific Cell Extraction Buffer (Cat No FNN0011) and the Coomassie Plus (Bradford) Assay kit were obtained from Invitrogen (USA).

\section{LX-2 culture}

LX-2 cells were cultured and maintained at $37^{\circ} \mathrm{C}$ in $5 \% \mathrm{CO}_{2}$ in DMEM high glucose supplemented media containing $10 \%$ heat-inactivated FBS, $10 \mathrm{U} / \mathrm{L}$ penicillin, $100 \mu \mathrm{g} / \mathrm{mL}$ streptomycin, and $2.5 \mu \mathrm{g} / \mathrm{mL}$ Fungizone.

\section{Cell treatments}

Cells were divided into five groups and seeded at $2 \times 10^{6}$ each in $10-\mathrm{cm}$ culture dishes. Two groups comprised the untreated cells (medium with DMSO only), whereas the other three groups were subjected to various treatments for $24 \mathrm{~h}$. To the first of the treated groups of cells, acetaldehyde $(100 \mu \mathrm{M})$ was applied; to the second and third groups of treated cells, acetaldehyde $(100 \mu \mathrm{M})$ plus alpha-mangostin $(10 \mu \mathrm{M})$ and acetaldehyde $(100 \mu \mathrm{M})$ plus alpha-mangostin $(20 \mu \mathrm{M})$ were applied, respectively. To the fifth group, only alpha-mangostin $(10 \mu \mathrm{M})$ was 


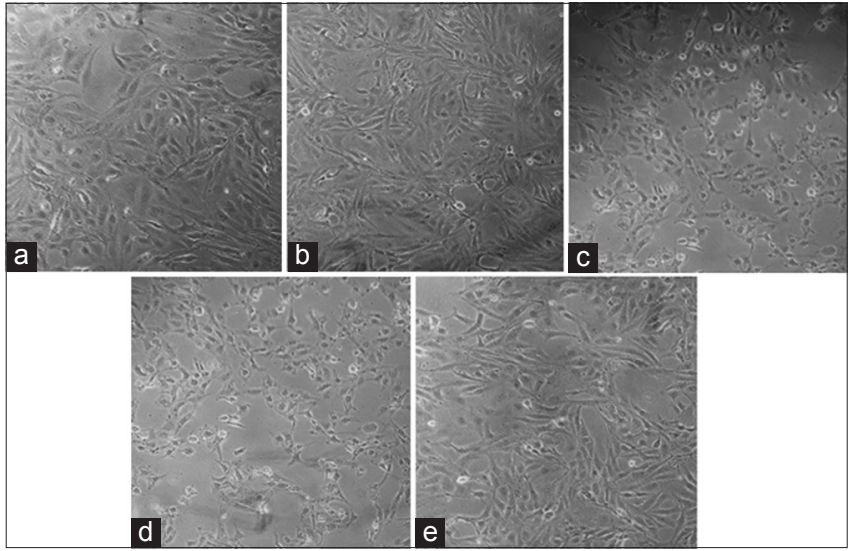

Fig. 1: Treatment of acetaldehyde-induced LX-2 cells with alpha-mangostin. Following $48 \mathrm{~h}$ of treatment, morphological observation of the LX-2 cells was performed with an inverted microscope. The treated LX- 2 cells did not exhibit any morphological changes (10× magnification). (a) Control group

(normal media only), (b) $100 \mu \mathrm{M}$ acetaldehyde induction, (c) treatment with $10 \mu \mathrm{M}$ alpha-mangostin and induction with $100 \mu \mathrm{M}$ acetaldehyde, (d) treatment with $20 \mu \mathrm{M}$ alpha-mangostin and induction with $100 \mu \mathrm{M}$ acetaldehyde, and (e) treatment with $10 \mu \mathrm{M}$ alpha-mangostin (no acetaldehyde induction)

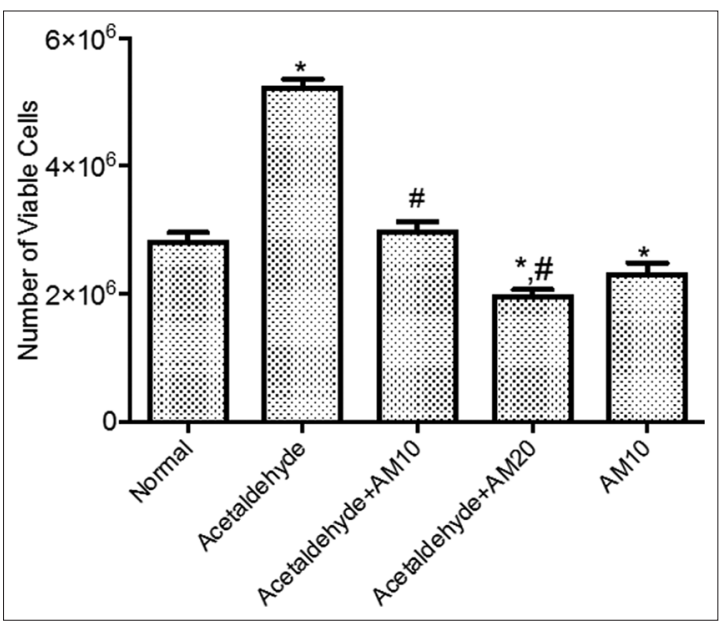

Fig. 2: Effect of alpha-mangostin on hepatic stellate cells proliferation. Cell viability of the LX- 2 cells was determined after induction with acetaldehyde and treatment with alphamangostin. Normal: Control group; Acetaldehyde: Induced with $100 \mu \mathrm{M}$ acetaldehyde; Acetaldehyde+AM10: Treated with $10 \mu \mathrm{M}$ alpha-mangostin and induced with $100 \mu \mathrm{M}$ acetaldehyde; Acetaldehyde+AM20: Treated with $20 \mu \mathrm{M}$ alpha-mangostin and induced with $100 \mu \mathrm{M}$ acetaldehyde; AM 10: Treatment with $10 \mu \mathrm{M}$ alpha-mangostin only (no induction). Results are presented as the mean $\pm S E$. ${ }^{*} p<0.05$ versus normal group; $\# p<0.05$ versus acetaldehyde group

applied. After $24 \mathrm{~h}$ of treatment, the cells were harvested and subjected to analysis of cell viability, and the proteins were extracted for antioxidant analysis. Cell viability was examined with the trypan blue exclusion method. All experiments were performed in triplicate.

\section{Spectrophotometry analysis}

The malondialdehyde (MDA) levels in the cell medium were determined in a microplate reader using colorimetric method described by Siddique et al. [13], while glutathione (GSH) levels were measured using Reduced GSH Assay Kit (Elabscience, USA). Activity of superoxide dismutase

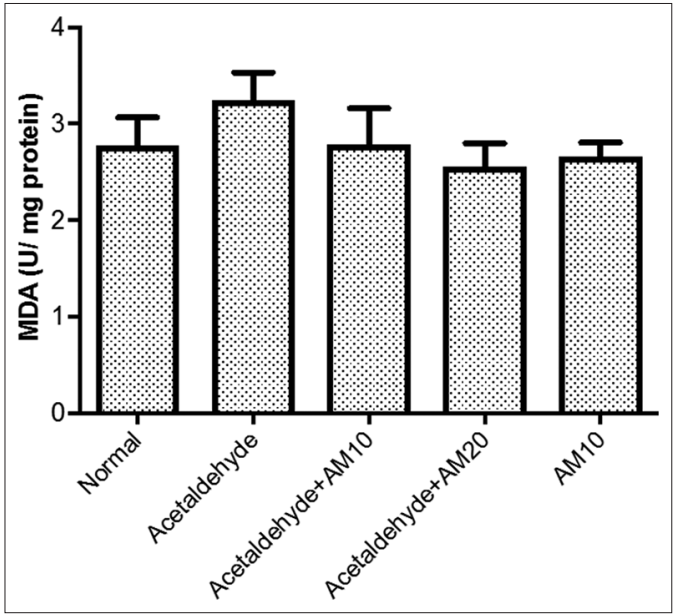

Fig. 3: Effect of alpha-mangostin on malondialdehyde (MDA) levels. The MDA level in LX-2 cells was determined after acetaldehyde induction and treatment with alpha-mangostin. Normal: Control group; Acetaldehyde: Induced with $100 \mu \mathrm{M}$ acetaldehyde; Acetaldehyde+AM10: Treated with $10 \mu \mathrm{M}$ alpha-mangostin and induced with $100 \mu \mathrm{M}$ acetaldehyde; Acetaldehyde+AM20: Treated with $20 \mu \mathrm{M}$ alpha-mangostin and induced with $100 \mu \mathrm{M}$ acetaldehyde; AM 10: Treatment with $10 \mu \mathrm{M}$ alpha-mangostin only (no induction). Results are presented as the mean \pm Standard error of the mean

(SOD) in cell lysates was measured using the method of Misra and Fridovich [14].

Statistical analysis

Results are presented as the mean \pm Standard error of the mean. Statistical analysis was performed with one-way analysis of variance. All values with $\mathrm{p}<0.05$ were considered statistically significant.

\section{RESULTS}

\section{Cell morphology}

After $48 \mathrm{~h}$ of treatment of the acetaldehyde-induced LX-2 cells with or without alpha-mangostin, no changes in morphology were observed under an inverted microscope (Fig. 1).

\section{Cell viability}

Acetaldehyde induction at $100 \mu \mathrm{M}$ resulted in a significant increase $(\mathrm{p}<0.05)$ in LX-2 proliferation compared with the control group (Fig. 2). In contrast, alpha-mangostin treatment significantly suppressed $(p<0.05)$ acetaldehyde-induced LX-2 proliferation in a dose-dependent manner.

Alpha-mangostin restores MDA levels in the LX-2 culture medium after acetaldehyde induction

Induction with $100 \mu \mathrm{M}$ acetaldehyde slightly increased MDA levels in the LX-2 culture medium compared with the control group, whereas treatment with alpha-mangostin restores MDA accumulation (Fig. 3).

\section{Alpha-mangostin significantly increased GSH levels}

Induction with $100 \mu \mathrm{M}$ acetaldehyde significantly decreased the GSH levels $(p<0.05)$ and SOD activity (not statistically significant) of LX-2 cells compared with the control group, whereas alpha-mangostin significantly enhanced $(\mathrm{p}<0.05)$ the GSH level (Fig. 4).

\section{DISCUSSION}

In this study, no change was observed in the morphology of the LX-2 cells between the control and treatment groups because the cells were already activated with a myofibroblast-like phenotype. Acetaldehyde was, however, observed to enhance HSC proliferation by increasing 


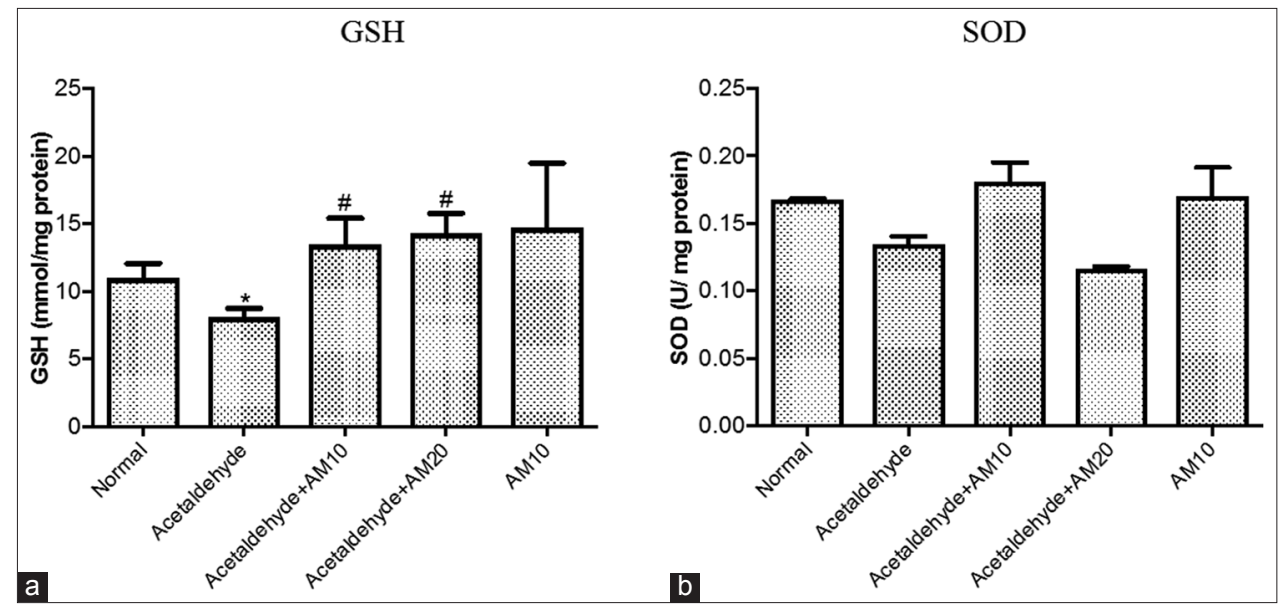

Fig. 4: Effect of alpha-mangostin on antioxidant activity. The glutathione levels (a) and superoxide dismutase activity (b) of LX-2 cells were determined after induction with acetaldehyde and treatment with alpha-mangostin. Normal: Control group; Acetaldehyde: Induced with $100 \mu \mathrm{M}$ acetaldehyde; Acetaldehyde+AM10: Treated with $10 \mu \mathrm{M}$ alpha-mangostin and induced with $100 \mu \mathrm{M}$ acetaldehyde; Acetaldehyde+AM20: Treated with $20 \mu \mathrm{M}$ alpha-mangostin and induced with $100 \mu \mathrm{M}$ acetaldehyde; AM 10: Treatment with $10 \mu \mathrm{M}$ alphamangostin only without acetaldehyde induction. Results are presented as the mean $\pm S E$. ${ }^{*} p<0.05$ versus normal group; \#p<0.05 versus acetaldehyde group

cell viability. This result was in line with research conducted by Wang et al. and Zhang et al., which showed that acetaldehyde significantly increases HSC viability $[15,16]$.

Acetaldehyde was known to elevate intracellular HSC ROS production and oxidative stress which leads to HSC activation promoting fibrogenesis $[8,9]$. The effects of acetaldehyde on the oxidative stress of HSC cells were studied in this research. Exposure to acetaldehyde caused an increase in the production of ROS in the HSCs, as indicated by a decrease in the concentration of GSH and the activity of SOD, both of which are important in the detoxification of ROS [17]. The effect of alpha-mangostin activity on these processes was then analyzed at two concentrations ( 10 and $20 \mu \mathrm{M}$ ), and this compound was found to significantly increase the levels of GSH. These concentrations of alphamangostin were chosen on the basis of previous research conducted by Rahmaniah et al., who reported a 50\% cytotoxic concentration for alpha-mangostin in LX-2 cells of $10.77 \mu \mathrm{M}$ [12].

MDA is a metabolite formed by lipid peroxidation of the cell membrane. Increased levels of MDA indicate the presence of lipid damage to the cell membrane due to oxidative stress [18]. MDA is, therefore, generally considered to be a marker of intracellular oxidative stress and an indicator of lipid peroxidation [17]. In this study, MDA levels were observed to increase in the acetaldehyde-induced group. On the other hand, alpha-mangostin antioxidant activity was expected to prevent this elevation; although there was no significant difference, alphamangostin did reduce the MDA levels in a dose-dependent manner.

GSH is an intracellular antioxidant that helps mitigate the oxidative stress caused by various ROS sources. Decreased cellular GSH levels indicate that the cell is experiencing oxidative stress caused by ROS, which requires neutralization by GSH. SOD is an antioxidant enzyme that protects cells from oxidative stress by catalyzing the process of superoxide dismutation into oxygen and hydrogen peroxide. Decreased SOD activity indicates the presence of oxidative stress caused by superoxide [19]. In this study, the antioxidant activity of alphamangostin was illustrated by the prevention of cellular GSH loss and increased SOD activity.

The use of antioxidants alone for the treatment of liver disease caused by alcohol is still controversial. The results of clinical trials indicate that antioxidants can be used for prevention but are not effective for the treatment of alcohol-related liver fibrosis [20]. The mechanisms and effects of acetaldehyde induction should be further assessed in future studies by examining changes in specific biomarkers. Further, research is also required to elucidate the mechanisms underlying the activity of alpha-mangostin and to develop its potential as an antifibrotic.

\section{CONCLUSION}

Alpha-mangostin was shown to normalize cell viability in HSCs induced by acetaldehyde, which at least in part resulted from protective effect of alpha-mangostin in preventing the loss of intracellular GSH.

\section{ACKNOWLEDGMENT}

This study was supported by a Universitas Indonesia International Publication Grant

\section{CONFLICTS OF INTEREST}

The authors state that there are no conflicts of interest regarding publication of this article.

\section{REFERENCES}

1. Chrostek L, Panasiuk A. Liver fibrosis markers in alcoholic liver disease. World J Gastroenterol 2014;20:8018-23.

2. Sidharthan S, Kottilil S. Mechanisms of alcohol-induced hepatocellular carcinoma. Hepatol Int 2014;8:452-7.

3. Hernandez-Gea V, Friedman SL. Pathogenesis of liver fibrosis. Annu Rev Pathol Mech Dis 2011;6:425-56.

4. Koyama Y, Brenner DA. New therapies for hepatic fibrosis. Clin Res Hepatol Gastroenterol 2015;39 Suppl 1:S75-9.

5. Li JT, Liao ZX, Ping J, Xu D, Wang H. Molecular mechanism of hepatic stellate cell activation and antifibrotic therapeutic strategies. J Gastroenterol 2008;43:419-28.

6. Wang JH, Batey RG, George J. Role of ethanol in the regulation of hepatic stellate cell function. World J Gastroenterol 2006;12:6926-32.

7. Setshedi M, Wands JR, de la Monte SM. Acetaldehyde adducts in alcoholic liver disease. Oxid Med Cell Longev 2010;3:178-85.

8. Liu R-M, Desai LP. Reciprocal regulation of TGF- $\beta$ and reactive oxygen species: A perverse cycle for fibrosis. Redox Biol 2015;6:565-77.

9. Mittal M, Siddiqui MR, Tran K, Reddy SP, Malik AB. Reactive oxygen species in inflammation and tissue injury. Antioxid Redox Signal 2014:20:1126-67.

10. Ibrahim MY, Hashim NM, Mariod AA, Mohan S, Abdulla MA, Abdelwahab SI, et al. $\alpha$-mangostin from Garcinia mangostana Linn: An updated review of its pharmacological properties. Arab J Chem 2016;9:317-29

11. Lee LT, Liu SH, Lin MN, Hu NY, Tsai YF, Shih YC, et al. Effects of mangosteen on $\alpha$-SMA expression in HSC-T6 cells and liver fibrosis in rats. J Chin Med 2013;24:211-22 
12. Rahmaniah R, Yuyuntia Y, Soetikno V, Arozal W, Antarianto R, Louisa M. Alpha mangostin inhibits hepatic stellate cells activation through TGF- $\beta$ /Smad and Akt signaling pathways: An in vitro study in LX2. Drug Res 2018;68:153-8.

13. Siddique YH, Ara G, Afzal M. Estimation of lipid peroxidation induced by hydrogen peroxide in cultured human lymphocytes. Dose Response 2012;10:1-0.

14. Misra HP, Fridovich I. The role of superoxide anion in the autooxidation of epinephrine and a simple assay for superoxide dismutase. J Biol Chem 1972;247:3170-5

15. Wang H, Guan W, Yang W, Wang Q, Zhao H, Yang F, et al. Caffeine inhibits the activation of hepatic stellate cells induced by acetaldehyde via adenosine A2A receptor mediated by the cAMP/PKA/SRC/ ERK1/2/P38 MAPK signal pathway. PLoS One 2014;9:e92482.
16. Zhang QS, Luk JM, Zhang J, Tian GY. Targeting glycyrrhetinic acid to hepatic stellate cells in treating rat liver fibrosis. Zhonghua Gan Zang Bing Za Zhi 2005;13:664-7.

17. Yan T, Zhao Y, Zhang X. Acetaldehyde induces cytotoxicity of SHSY5Y cells via inhibition of akt activation and induction of oxidative stress. Oxid Med Cell Longev 2016;2016:4512309.

18. Ayala A, Muñoz MF, Argüelles S. Lipid peroxidation: Production, metabolism, and signaling mechanisms of malondialdehyde and 4-hydroxy-2-nonenal. Oxid Med Cell Longev 2014;2014:360438.

19. Birben E, Sahiner UM, Sackesen C, Erzurum S, Kalayci O. Oxidative stress and antioxidant defense. World Allergy Organ J 2012;5:9-19.

20. Han KH, Hashimoto N, Fukushima M. Relationships among alcoholic liver disease, antioxidants, and antioxidant enzymes. World J Gastroenterol 2016;22:37-49. 